\title{
Nonnegative Diffusion Orientation Distribution Function
}

\section{Liqun Qi, Gaohang Yu \& Yi Xu}

\section{Journal of Mathematical Imaging and} Vision

\section{ISSN 0924-9907}

Volume 45

Number 2

J Math Imaging Vis (2013) 45:103-113

DOI 10.1007/s10851-012-0346-y

\section{JOURNAL OF MATHEMATICAL} IMAGING AND VISION

Founding Editor:

Gerhard X. Ritter

University of Florida

Editor-in-Chief:

Joachim Weikert

Saarland University

Associate Editor-in-Chief:

Selim Esedoghe

University of Michigan

Springer 
Your article is protected by copyright and all rights are held exclusively by Springer Science+Business Media, LLC. This e-offprint is for personal use only and shall not be selfarchived in electronic repositories. If you wish to self-archive your work, please use the accepted author's version for posting to your own website or your institution's repository. You may further deposit the accepted author's version on a funder's repository at a funder's request, provided it is not made publicly available until 12 months after publication. 


\title{
Nonnegative Diffusion Orientation Distribution Function
}

\author{
Liqun Qi • Gaohang Yu • Yi Xu
}

Published online: 31 May 2012

(C) Springer Science+Business Media, LLC 2012

\begin{abstract}
Because of the well-known limitations of diffusion tensor imaging (DTI) in regions of low anisotropy and multiple fiber crossing, high angular resolution diffusion imaging (HARDI) and Q-Ball Imaging (QBI) are used to estimate the probability density function (PDF) of the average spin displacement of water molecules. In particular, QBI is used to obtain the diffusion orientation distribution function (ODF) of these multiple fiber crossing. As a probability distribution function, the orientation distribution function should be nonnegative which is not guaranteed in the existing methods. This paper proposes a novel technique to guarantee the nonnegative property of ODF by solving a convex optimization problem, which has a convex quadratic objective function and a constraint involving the nonnegativity requirement on the smallest $Z$-eigenvalue of the diffusivity tensor. Using convex analysis and optimization techniques, we first derive the optimality conditions of this convex optimization problem. Then, we propose a gradient descent algorithm to solve this problem. We also present formulas for determining the principal directions (maxima) of the ODF. Numerical examples on synthetic data as well as
\end{abstract}

\section{Qi · G. Yu $(\bowtie) \cdot$ Y. Xu}

Department of Applied Mathematics, The Hong Kong

Polytechnic University, Hung Hom, Kowloon, Hong Kong

e-mail: maghyu@163.com

L. Qi

e-mail: maqilq@inet.polyu.edu.hk

Y. Xu

e-mail: yi.xu1983@gmail.com

G. $\mathrm{Yu}$

Jiangxi Key Laboratory of Numerical Simulation Technology,

School of Mathematics and Computer Sciences, Gannan Normal

University, Ganzhou 341000, China
MRI data are displayed to demonstrate the significance of our approach.

Keywords High angular resolution diffusion imaging (HARDI) · Orientation distribution function (ODF) . Nonnegativity $\cdot$ Higher order diffusion tensor $\cdot$ Principal direction

\section{Introduction}

It is well-known that the popular magnetic resonance imaging (MRI) model, the diffusion tensor imaging model (DTI) [4-6] breaks down in regions of low anisotropy and multiple fiber crossing. In order to overcome this drawback of DTI, Tuch et al. [28] proposed a novel approach, high angular resolution diffusion imaging (HARDI) in 2002. In 2004, Tuch [27] further introduced Q-ball imaging (QBI) to reconstruct the diffusion orientation distribution function (ODF) of the underlying fiber population of a biological tissue.

The ODF is a function on the unit sphere describing the probability averaged over the voxel that a particle will diffuse into any solid angle. As the water molecules in normal tissues tend to diffuse along fibers when contained in fiber bundles [6], the principal directions (maxima) of the ODF agree with the true synthetic fiber directions. Tuch [27] showed that the ODF can be estimated directly from the raw HARDI signal on a single sphere of q-space by the FunkRadon transformation. This is the main idea of QBI.

The Funk-Radon transformation involves integral with the Dirac delta function. In [11], Descoteax et al. suggested to use the Funk-Hecke theorem and higher order spherical harmonics to obtain a mathematical simplification of the Funk-Radon transformation. It was shown that the ODF estimation in [11] is up to 15 times faster than the numerical method in [27]. 
In [10], Descoteax et al. showed that both spherical harmonics and higher order tensors restricted to the unit sphere forms a basis of the same functional space. Ghosh et al. [14] presented a polynomial based approach to extract maxima of the orientation distribution function in diffusion MRI. In [7], using the Z-eigenvalue concept introduced in [21], Bloy and Verma suggested to determine the principal directions (maxima) of the ODF by a curvature concept they introduced. For an excellent recent survey of higher order diffusion tensor methods in diffusion MRI, see Ghosh and Deriche [13].

In the study of the apparent diffusion coefficient (ADC) profile, in 2003, Ozarslan and Mareci [18] proposed to model the ADC profile with higher order diffusion tensors (HODT), that can reflect more complex micro-geometries of biological tissues. An intrinsic property of the diffusivity profile is positive semi-definite [2, 3, 8, 10, 15, 30]. In [25], we approximate the ADC profile by a positive semi-definite diffusion tensor of either second or higher order and further show that by regarding a higher order tensor as a vector, the diffusivity function can be simply written as the inner product of two vectors. Such a observation provides additional mathematical tools and further information about the diffusivity function. For example, in [25], we use this observation to derive the subgradients of the smallest $\mathrm{Z}$-eigenvalue function of the diffusivity function, which is a measure of the positive definiteness of the diffusivity function.

The ODF, as a probability distribution function, should be nonnegative. Until now, no ODF models have addressed this. Recently, Tournier et al. [29] proposed a nonnegativity penalty fiber orientation distribution (FOD) model by penalizing FOD values. That model does not completely forbid negative FOD values. In this paper, we present a nonnegative ODF model. The ODF values are strictly nonnegative in our model. This is different from [29].

In the next section, we first show that there is a constant linear transformation relation between the vector versions of the raw HARDI signal and the ODF in the homogeneous polynomial basis. Such a linear transformation connection between the HARDI signal and the ODF not only saves the computational time, but also makes the nonnegative ODF model possible. Then, we propose a new model which can guarantee the nonnegative feature of the ODF function.

In Sect. 3 we present a new way on how to determine the principal directions (maxima) of the ODF, based on optimization theory, which is more precise. Numerical examples on synthetic data as well as MRI data are displayed in Sect. 4 to demonstrate our approach. Section 5 is a conclusion section.

\section{Nonnegative Diffusion Orientation Distribution Function}

Suppose that we use an $m$ th order symmetric tensor $\mathcal{A}$ to denote the raw HARDI signal $S$. Here $m$ is an even number as the signal is antipodally symmetric. Let $\mathbf{x}=\left(x_{1}, x_{2}, x_{3}\right)$ be a unit direction. Then the HARDI signal at the direction $\mathbf{x}$ takes the value

$S(\mathbf{x})=\sum_{i=0}^{m} \sum_{j=0}^{m-i} a_{i j} x_{1}^{i} x_{2}^{j} x_{3}^{m-i-j}$,

where $a_{i j}$ are the independent components of the tensor $\mathcal{A}$. Obviously, there are

$n=\sum_{i=1}^{m+1} i=\frac{1}{2}(m+1)(m+2)$

independent components $a_{i j}$. Let $k=j+1+i(2 m+3-$ $i) / 2, s_{k}=a_{i j}$ and $\hat{x}_{k}=x_{1}^{i} x_{2}^{j} x_{3}^{m-i-j}$. Then we can simplify (1) as

$S(\mathbf{x})=s^{\top} \hat{x}$,

i.e., we can regard $S(\mathbf{x})$ as the scalar product of vectors $s$ and $\hat{x}$ in $\Re^{n}$. This point of view will be useful for our analysis later on.

Similarly, we use an $m$ th order symmetric tensor $\mathcal{B}$ to denote the ODF $\Psi$. Let $\mathbf{x}=\left(x_{1}, x_{2}, x_{3}\right)$ be a unit direction in the q-space. Then the ODF value at the direction $\mathbf{x}$ has the value

$\Psi(\mathbf{x})=\sum_{i=0}^{m} \sum_{j=0}^{m-i} b_{i j} x_{1}^{i} x_{2}^{j} x_{3}^{m-i-j} \triangleq u^{\top} \hat{x}$,

where $b_{i j}$ denote the independent components of the tensor $\mathcal{B}$ and $u=\left(u_{1}, \ldots, u_{k}, \ldots, u_{n}\right)^{\top}$ is a vector in $\Re^{n}$ with $u_{k}=$ $b_{i j}$.

Below, we recall some basic facts about the spherical harmonics. Let $\Omega$ be the sphere in $\mathbb{R}^{3}$, that is,

$\Omega=\left\{\mathbf{x} \in \mathfrak{R}^{3}: x_{1}^{2}+x_{2}^{2}+x_{3}^{2}=1\right\}$.

We also use spherical co-ordinates $(\theta, \phi), 0 \leq \theta \leq \pi, 0 \leq$ $\phi \leq 2 \pi$, with

$\mathbf{x}=\left(\begin{array}{l}x_{1} \\ x_{2} \\ x_{3}\end{array}\right)=\left(\begin{array}{c}\sin \theta \cos \phi \\ \sin \theta \sin \phi \\ \cos \theta\end{array}\right)$.

Let $Y_{l}^{q}$ denote the spherical harmonics (SH) of order $l$ and degree $q$. Mathematically, it is given as follows

$Y_{l}^{q}=\sqrt{\frac{2 l+1}{4 \pi} \cdot \frac{(l-q) !}{(l+q) !}} P_{l}^{q}(\cos \theta) e^{i q \phi}$,

where $P_{l}^{q}$ is an associated Legendre polynomials, which can be obtained analytically from the following set of equations, 


$$
\begin{aligned}
& P_{l}(x)=\frac{1}{2^{l} l !}\left(\frac{d}{d x}\right)^{l}\left(x^{2}-1\right)^{l}, \\
& P_{l}^{q}(x)=(-1)^{q}\left(1-x^{2}\right)^{\frac{q}{2}}\left(\frac{d}{d x}\right)^{q} P_{l}(x), \quad q \geq 0, \\
& P_{l}^{-q}(x)=(-1)^{q} \frac{(l-q) !}{(l+q) !} P_{l}^{q}(x) .
\end{aligned}
$$

Let $l=0,2,4, \ldots, m$ and $q=-l, \ldots, 0, \ldots, l$. A single index $p$ in terms of $l$ and $q$ is used so that $p \equiv p(l, q)=$ $\left(l^{2}+l+2\right) / 2+q$. Then $p=1, \ldots, n$. If $p=p(l, q)$, then define $l(p)=l$. Explicitly, if $\left(l^{2}-l+2\right) / 2 \leq p \leq\left(l^{2}+\right.$ $3 l+2) / 2$, then $l(p)=l$. Consequently, we have $l(1)=0$, $l(p)=2$ for $2 \leq p \leq 6, l(p)=4$ for $7 \leq p \leq 15$, so on.

As in $[7,10,11]$, the real spherical harmonics of order less than or equal to $m$, are

$$
R_{p}(\theta, \phi)= \begin{cases}\sqrt{2} \operatorname{Re}\left(Y_{l}^{|q|}\right), & \text { if }-l \leq q \leq 0, \\ \sqrt{2}(-1)^{q+1} \operatorname{Im}\left(Y_{l}^{q}\right), & \text { if } 0<q \leq l,\end{cases}
$$

for $p=1, \ldots, n$, where $\operatorname{Re}\left(Y_{l}^{q}\right)$ and $\operatorname{Im}\left(Y_{l}^{q}\right)$ represent the real and imaginary parts of $Y_{l}^{q}$ respectively. Thus, the HARDI signal $S$ can be described as

$$
S(\theta, \phi)=\sum_{p=1}^{n} c_{p} R_{p}(\theta, \phi)
$$

Moreover, Descoteaux et al. [11] showed that the ODF can be expressed as

$\Psi(\theta, \phi)=\sum_{p=1}^{n} \underbrace{2 \pi P_{l(p)}(0) c_{p}}_{c_{p}^{\prime}} R_{p}(\theta, \phi)$,

where $P_{l(p)}(0)$ is a Legendre polynomial with simple expression

$$
P_{l(p)}(0)=(-1)^{\frac{l(p)}{2}} \frac{3 \cdot 5 \cdots(l(p)-1)}{2 \cdot 4 \cdots l(p)} .
$$

This demonstrated that the ODF can be estimated by scaling of the HARDI signal's spherical harmonic coefficients.

It should be note that, as a probability distribution function, the ODF should be nonnegative over its entire domain. However, as pointed out in [19], this constraint has been more challenging to satisfy within the spherical harmonics framework. In the following theorem, we show that there is a constant linear transformation relation between the vector versions of the HARDI signal and the ODF in the homogeneous polynomial basis. Such a linear transformation connection between the HARDI signal and the ODF enable us to model the nonnegative feature of the ODF.

Theorem 1 For $p, k=1, \ldots, n$, let

$t_{p k}=\int_{\Omega} x_{1}^{i} x_{2}^{j} x_{3}^{m-i-j} R_{p}(\mathbf{x}) d \Omega$.

Then $T=\left(t_{p k}\right)$ is an $n \times n$ invertible matrix. Let $D$ be an $n \times n$ diagonal matrix with its diagonal elements as
$P_{l(1)}(0), \ldots, P_{l(n)}(0)$. Let $A=2 \pi T^{-1} D T$. Then we have $u=A s$.

Proof We note that, both the $m$ th-order tensor polynomials restricted to the sphere and the even order spherical harmonics up to order $m$, are basis for the same function space. So, for the vector version $s$ of a HARDI signal $S$, there exists a vector $c$ of spherical harmonic coefficients such that $c=T s$ (see [10] for details). Thus, $T$ is invertible. By [11], we have $\Psi(\mathbf{x})=\sum_{p=1}^{n} 2 \pi P_{l(p)}(0) c_{p} R_{p}(\mathbf{x})$

where $c_{p}, p=1, \ldots, n$ are the spherical harmonics series coefficients of $S(\mathbf{x})$. Hence, the spherical harmonics series coefficients of $\Psi(\mathbf{x})$ are $\psi_{p}=2 \pi P_{l(p)}(0) c_{p}$. Let $c$ and $\psi$ be vectors in $\mathfrak{R}^{n}$ with components $c_{p}$ and $\psi_{p}$ for $p=1, \ldots, n$, respectively. Then we have $c=T s$ and $\psi=T u$. Thus, $u=$ $2 \pi T^{-1} D T s=A s$. The proof is complete.

Suppose that HARDI samples in $N$ gradient directions $\left\{\mathbf{g}_{h}: \mathbf{g}_{h} \in \Omega, h=1, \ldots, N\right\}, N \gg n$, and the corresponding HARDI signals on these $N$ gradients are described by $\left\{d_{h}: h=1, \ldots, N\right\}$. Then $\left\{\hat{\mathbf{g}}_{h}: h=1, \ldots, N\right\}$ are $N$ vectors in $\Re^{n}$. We assume that $\left\{\hat{\mathbf{g}}_{h}: h=1, \ldots, N\right\}$ spans $\Re^{n}$, i.e., there are $n$ vectors among these $N$ vectors, which are linearly independent, or we say that $\left\{\hat{\mathbf{g}}_{h}: h=1, \ldots, N\right\}$ has rank $n$. We call this assumption the full rank assumption. This assumption is needed so that the $N$ gradient directions $\left\{\mathbf{g}_{h}: h=1, \ldots, N\right\}$ can reflect the signal $S(\mathbf{g})$ sufficiently. Let $C$ be an $n \times N$ matrix, whose column vectors are $\hat{\mathbf{g}}_{h}, h=1, \ldots, N$. Let $B=C C^{\top}$. Then $B$ is an $n \times n$ positive semi-definite symmetric matrix. Under the full rank assumption, $B$ is a positive definite symmetric matrix. We also let $\mathbf{d}$ be a vector in $\Re^{N}$, with components $\left\{d_{h}: h=1, \ldots, N\right\}$.

The least squares problem for finding an $m$ th order tensor $\mathcal{A}$ to reflect the signal $S(\mathbf{g})$ is to find $\bar{s} \in \mathfrak{R}^{n}$ such that

$F(\bar{s})=\min _{s \in \Re^{n}} F(s)$,

where

$F(s)=\sum_{h=1}^{N}\left(S\left(\mathbf{g}_{h}\right)-d_{h}\right)^{2}=\sum_{h=1}^{N}\left(s^{\top} \hat{\mathbf{g}}_{h}-d_{h}\right)^{2}$.

It is well-known that under the full rank assumption the solution of the least squares problem (3) is

$\bar{s}=B^{-1} C d$.

The function $F$ is a convex quadratic function. To see this, by (4), for any $s \in \Re^{n}$, we can represent $F$ as

$F(s)=(s-\bar{s})^{\top} B(s-\bar{s})$.

Thus, $F$ is a convex quadratic function.

With $u=A s$, given by Theorem 1 , we have ODF $\Psi(\mathbf{x})=$ $u^{T} \hat{x}$. However, in this way, we cannot guarantee $\Psi(\mathbf{x}) \geq 0$ 
for all $\mathbf{x} \in \Omega$. In fact, $\Psi(\mathbf{x}) \geq 0$ for all $\mathbf{x}$ if and only if $u$ is positive semi-definite in the sense of [25]. Thus, motivated from [25], we formulate a new model as follows:

$$
F\left(s^{*}\right)=\min \left\{F(s): \lambda_{\min }(A s) \geq 0\right\} .
$$

Here, $\lambda_{\min }(A s)$ is a measure of positive definiteness of $u=$ As (see [25] for more discussions) given by

$\lambda_{\min }(u)=\min \left\{u^{T} \hat{x}: x_{1}^{2}+x_{2}^{2}+x_{3}^{2}=1\right\}$.

A possible method for solving (7) was given in [25]. For completeness purpose, we include it in Appendix.

We now have the following theorem:

Theorem $2 \lambda_{\min }(A s)$ is a continuous concave function of $s$. Hence, (6) is a convex optimization problem.

If $\lambda_{\min }(A \bar{s}) \geq 0$, then $s^{*}=\bar{s}$ is a global minimizer of (6). If the full rank assumption holds, then (6) has a unique global minimizer.

Suppose that the full rank assumption holds and $\lambda_{\min }(A \bar{s})$ $<0$. Then, $s^{*}$ is the unique global minimizer of (6) if and only if there is a positive number $\mu$ such that

$\left\{\begin{array}{l}B\left(s^{*}-\bar{s}\right)=\mu A^{\top} \hat{\mathbf{x}}^{*}, \\ \lambda_{\min }\left(A s^{*}\right)=0,\end{array}\right.$

where $A^{\top} \hat{\mathbf{x}}^{*}$ is a subgradient [26] of the concave function $\lambda_{\min }$ at $s^{*}$. By (8), we have

$\left\{\begin{array}{l}\left(s^{*}\right)^{\top} B\left(s^{*}-\bar{s}\right)=0, \\ \left(\hat{\mathbf{x}}^{*}\right)^{\top} A s^{*}=0 .\end{array}\right.$

Proof Let $s^{(1)}, s^{(2)} \in \Re^{n}, 0 \leq t \leq 1$ and $s=t s^{(1)}+(1-$ t) $s^{(2)}$. Suppose $\mathbf{x}^{*}$ is a global minimizer of (7). Then $\left(x_{1}^{*}\right)^{2}+$ $\left(x_{2}^{*}\right)^{2}+\left(x_{3}^{*}\right)^{2}=1$ and

$$
\begin{aligned}
\lambda_{\min }(A s) & =\Psi\left(\mathbf{x}^{*}\right) \\
& =t\left(\hat{\mathbf{x}}^{*}\right)^{\top} A s^{(1)}+(1-t)\left(\hat{\mathbf{x}}^{*}\right)^{\top} A s^{(2)} \\
& \geq t \lambda_{\min }\left(A s^{(1)}\right)+(1-t) \lambda_{\min }\left(A s^{(2)}\right) .
\end{aligned}
$$

This shows that $\lambda_{\min }(A s)$ is a concave function of $s$. Since $\lambda_{\min }(A s)$ is a concave function defined in the whole space $\Re^{n}$, according to the theory of convex analysis [26], it is a continuous function. By (5), $F$ is a convex quadratic function of $s$. Hence, (7) is a convex optimization problem.

If $\lambda_{\min }(A \bar{s}) \geq 0$, then $\bar{s}$ satisfies the constraint of (6). Since $\nabla F(\bar{s})=0, s^{*}=\bar{s}$ is a global minimizer of (6). If the full rank assumption holds, then $F$ is strictly convex. Hence, (6) has a unique global minimizer in this case.

Suppose that the full rank assumption holds and $\lambda_{\min }(A \bar{s})$ $<0$. Then, the minimization problem (6), has a unique global minimizer $s^{*}$ which satisfies $\lambda_{\min }\left(A s^{*}\right) \geq 0$ and $F\left(s^{*}\right)>F(\bar{s})$. Suppose that $\lambda_{\min }\left(A s^{*}\right)>0$. Since $\lambda_{\min }(A s)$ is continuous, there is a $\tilde{s}$ in the segment connecting $s^{*}$ and $\bar{s}$ such that $\lambda_{\min }(A \tilde{s})=0$ and $F(\tilde{s})<F\left(s^{*}\right)$. This contradicts the fact that $s^{*}$ is a global minimizer of (6). Hence, we have $\lambda_{\min }\left(A s^{*}\right)=0$. Now, (8) follows from (5) and the optimality condition of the convex optimization problem (6). By (7), we have

$\lambda_{\min }\left(A s^{*}\right)=\Psi\left(\mathbf{x}^{*}\right)=\left(s^{*}\right)^{\top} A^{\top} \hat{\mathbf{x}}^{*}$.

From this and the second equation of (8), we have the second equation of (9). Taking inner product with $s^{*}$ on the two sides of the first equation of (8) and combining it with the second equation of (9), we have the first equation of (9).

Suppose that $\mathbf{x}$ is a global minimizer of (7). Since $\Psi(\mathbf{x})=$ $u^{\top} \hat{\mathbf{x}}$, we have

$\lambda_{\min }(A s)=\hat{\mathbf{x}}^{\top} A s$.

When $m$ is even, if $\mathbf{x}$ is a global minimizer of (7), then $\mathbf{y}=-\mathbf{x}$ is also a global minimizer of (7). However, we have $\hat{\mathbf{y}}=\hat{\mathbf{x}}$ in this case. Therefore, such $\hat{\mathbf{x}}$ in (10), generated by a global minimizer $\mathbf{x}$, may still be unique even if the global minimizers are not unique. Using standard convex analysis facts, we know that if such $\hat{\mathbf{x}}$ in (10) is unique, then $\lambda_{\min }(A s)$ is differentiable at $s$ and its gradient is given by $A^{\top} \hat{\mathbf{x}}$. On the other hand, if such $\hat{\mathbf{x}}$ is not unique, then any of such $A^{\top} \hat{\mathbf{x}}$ is a subgradient of $\lambda_{\min }(A s)$ at $s$ and the subdifferential of $\lambda_{\min }(A s)$ at $s$ is the convex hull of all such $A^{\top} \hat{\mathbf{x}}$. With such knowledge of gradients and subgradients of $\lambda_{\min }(A s)$, we can solve convex optimization problem (6) by a standard convex programming method [16].

Under the full rank assumption, we may use (4) to calculate $\bar{s}$. If $\lambda_{\min }(A \bar{s}) \geq 0$, then $s^{*}=\bar{s}$ and we have the solution needed. If $\lambda_{\min }(A \bar{s})<0$, by Theorem $2, \lambda_{\min }\left(A s^{*}\right)=0$. Hence, in this case, we only need to solve an equality constrained optimization problem. In the following table, we give a summary of the nonnegative ODF model where the analytical ODF was estimated in the homogeneous polynomial basis.

(1) Calculate $\bar{s}$ by (4). If $\lambda_{\min }(A \bar{s}) \geq 0$, then $s^{*}=\bar{s}$ and we have the solution needed.

(2) If $\lambda_{\min }(A \bar{s})<0$, by Theorem $2, \lambda_{\min }\left(A s^{*}\right)=0$. Hence, in this case, we only need to solve the following equality constrained optimization problem:

$$
F\left(s^{*}\right)=\min \left\{F(s): \lambda_{\min }(A s)=0\right\} .
$$

(3) Then we have $u=A s^{*}$ and the nonnegative $\operatorname{ODF} \Psi(\mathbf{x})=u^{\top} \hat{\mathbf{x}}$.

Clearly, problem (11) still has (8) as its optimality condition under the full rank assumption. We may consider to solve this equality constrained problem by some standard minimization methods [17].

Generally, the diffusion peaks of the ODF are small and so direct visualization of the ODF often shows little orientational structure. So, it will be helpful to sharpen or min-max 
normalize the ODF [11, 27]. In [11], Descoteaux et al. proposed a fast and robust algorithm to estimate ODF incorporating a sharpening scheme based on the Laplace-Beltrami operator. Let $d: \mathfrak{R}^{3} \rightarrow \mathfrak{R}^{N}$ be the vector field of diffusion signal in $N$ discrete directions on the sphere. Let $\widetilde{C}$ be an $n \times N$ matrix, whose column vectors are SH functions $R_{p}$. Let $\widetilde{B}=\widetilde{C} \widetilde{C}^{\top}$ and $L$ be an $n \times n$ diagonal matrix with its diagonal elements as $L_{i, i}=\left(l(i)^{2}(l(i)+1)^{2}\right)$. Then, their ODF estimation is expressed using the spherical harmonic basis

$\Psi(\mathbf{x})=\sum_{p=1}^{n} 2 \pi P_{l(p)}(0) \bar{c}_{p} R_{p}(\mathbf{x})$,

where the spherical harmonics series coefficients $\bar{c}_{p}$ can be obtained by a modified Moore-Penrose pseudo-inverse scheme

$\bar{c}=(\widetilde{B}+\alpha L)^{-1} \widetilde{C} d$.

Here $\alpha$ is the weight term on the Laplace-Beltrami smoothing matrix $L$, which penalizes high degrees in the estimation under the assumption that high degrees $\mathrm{SH}$ are likely to capture noise.

Let $\widetilde{A}=2 \pi T^{-1} D$ and $u=\widetilde{A} \bar{c}$. Then, the ODF can be represented by a real homogeneous polynomial of order $m$ in the form that $\Psi(\mathbf{x})=u^{\top} \hat{\mathbf{x}}$. In order to satisfy the nonnegative constraint, one can solve the following model:

$\min \left\{\bar{F}(c): \lambda_{\min }(\widetilde{A} c) \geq 0\right\}$,

where $\bar{F}$ denotes a convex quadratic function

$\bar{F}(c)=(c-\bar{c})^{\top}(\widetilde{B}+\alpha L)(c-\bar{c})$.

Similarly, we have the following theorem.

Theorem $3 \lambda_{\min }(\widetilde{A} c)$ is a continuous concave function of $c$. Hence, (13) is a convex optimization problem.

If $\lambda_{\min }(\widetilde{A} \bar{c}) \geq 0$, then $c^{*}=\bar{c}$ is a global minimizer of (13).

Suppose that $\lambda_{\min }(\widetilde{A} \bar{c})<0$. Then, $c^{*}$ is the unique global minimizer of (13) if and only if there is a positive number $\mu$ such that

$\left\{\begin{array}{l}(\widetilde{B}+\alpha L)\left(c^{*}-\bar{c}\right)=\mu(\widetilde{A})^{\top} \hat{\mathbf{x}}^{*}, \\ \lambda_{\min }\left(\widetilde{A} c^{*}\right)=0\end{array}\right.$

where $(\widetilde{A})^{\top} \hat{\mathbf{x}}^{*}$ is a subgradient of the concave function $\lambda_{\min }$ at $c^{*}$. Moreover, by (14), we have

$\left\{\begin{array}{l}\left(c^{*}\right)^{\top}(\widetilde{B}+\alpha L)\left(c^{*}-\bar{c}\right)=0, \\ \left(\hat{\mathbf{x}}^{*}\right)^{\top} \widetilde{A} c^{*}=0 .\end{array}\right.$

The main contribution of the work presented here is a nonnegative ODF model with a sharpening scheme. We give a summary as follows.
- Calculate $\bar{c}$ by (12). If $\lambda_{\min }(\widetilde{A} \bar{c}) \geq 0$, then we have the solution needed $c^{*}=\bar{c}$.

- Else if $\lambda_{\min }(\widetilde{A} \bar{c})<0$, we need to solve (13) or the following equality constrained optimization problem:

$\bar{F}\left(c^{*}\right)=\min \left\{\bar{F}(c): \lambda_{\min }(\widetilde{A} c)=0\right\}$.

- Then we have $u=\widetilde{A} c^{*}$ and the nonnegative ODF $\Psi(\mathbf{x})=u^{\top} \hat{\mathbf{x}}$.

Let $\mathcal{P}^{+}$denote a projection operator which projects a tensor onto the positive semi-definite cone. An approximate projected gradient algorithm for solving nonnegative ODF model can be presented as follows.

\section{Algorithm PSD-ODF}

Data. Given a constant $\varepsilon>0$. Calculate $\bar{c}$ by (12) and then get an initial guess $\bar{u}=\widetilde{A} \bar{c}$. If $\lambda_{\min }(\bar{u}) \geq 0$, Stop; Otherwise set $u_{1}=\mathcal{P}^{+}(\bar{u})$.

Initialization. Set $s_{1}=-(\widetilde{B}+\alpha L)\left(u_{1}-\bar{u}\right)$ and $k=1$.

While $\left|u_{k}^{\top}(\widetilde{B}+\alpha L)\left(u_{k}-\bar{u}\right)\right|>\varepsilon$ or $\lambda_{\min }\left(u_{k}\right)>\varepsilon$ do

Compute $t_{k}$ such that $u_{k+1}:=u_{k}+t_{k} s_{k}$ satisfies $u_{k+1}^{\top}(\widetilde{B}+$ $\alpha L)\left(u_{k+1}-\bar{u}\right)=0$;

Compute $\lambda_{\min }\left(u_{k+1}\right)$. if $\lambda_{\min }\left(u_{k+1}\right)<0$, then set $u_{k+1}=$ $\mathcal{P}^{+}\left(u_{k+1}\right)$.

Compute $s_{k+1}=-(\widetilde{B}+\alpha L)\left(u_{k+1}-\bar{u}\right)$ and set $k=k+1$.

End while.

One of the advantages of the homogeneous polynomial basis over the spherical harmonic basis is that the local maxima of the ODF can be easily computed. In the next section, we present formulas for determining the principal directions of the ODF.

\section{Principal Directions of ODF}

Recently, using the Z-eigenvalue concept introduced in [21], Bloy and Verma [7] suggested to determine the principal directions (maxima) of the ODF by the curvature concept they introduced. In contrast, in this section, we use optimization theory and techniques to determine the principal directions (maxima) of the ODF.

After a nonnegative ODF $\Psi$ is found, we can solve

$\max \left\{\Psi(\mathbf{x}): x_{1}^{2}+x_{2}^{2}+x_{3}^{2}=1\right\}$.

The optimization conditions of (16) are (18), the same as the optimality conditions of (7). Actually, in Appendix, we give the method to calculate all the Z-eigenvalues [21] of $u$. The largest $Z$-eigenvalue $\lambda_{\max }$ gives the global maximum value of $u$. Then, the corresponding solution $\mathbf{x}$, which is called the Z-eigenvector of $u$, associated with $\lambda_{\max }$, is the leading principal direction of $u$. The Z-eigenvectors of $u$, associated 
with the other Z-eigenvalues, include local maximizers, local minimizers and saddle points. Suppose that $(\mathbf{x}, \lambda)$ is a solution of (18). Then $\lambda$ is a Z-eigenvalue of $u, x$ is a stationary point of (16).

The Hessian of the Lagrangian function of $(18)$ at $(\mathbf{x}, \lambda)$ is given by

$\nabla_{\mathbf{x}}^{2} L(\mathbf{x}, \lambda)=R-m(m-1) \lambda I$,

where $I$ is the $3 \times 3$ unit matrix and $R=\left(r_{i j}\right)$ is a $3 \times 3$ symmetric matrix. Denotes $m(i, j)=m-i-j$, then

$$
\begin{aligned}
& r_{11}=\sum_{i=2}^{m} \sum_{j=0}^{m-i} i(i-1) b_{i j} x_{1}^{i-2} x_{2}^{j} x_{3}^{m(i, j)}, \\
& r_{12}=\sum_{i=1}^{m} \sum_{j=1}^{m-i} i j b_{i j} x_{1}^{i-1} x_{2}^{j-1} x_{3}^{m(i, j)}, \\
& r_{13}=\sum_{i=1}^{m} \sum_{j=0}^{m-i-1} i m(i, j) b_{i j} x_{1}^{i-1} x_{2}^{j} x_{3}^{m(i, j)-1}, \\
& r_{22}=\sum_{i=0}^{m} \sum_{j=2}^{m-i} j(j-1) b_{i j} x_{1}^{i} x_{2}^{j-2} x_{3}^{m(i, j)}, \\
& r_{23}=\sum_{i=0}^{m} \sum_{j=1}^{m-i-1} j m(i, j) b_{i j} x_{1}^{i} x_{2}^{j-1} x_{3}^{m(i, j)-1}, \\
& r_{33}=\sum_{i=0}^{m} \sum_{j=0}^{m-i-2} m(i, j)(m(i, j)-1) b_{i j} x_{1}^{i} x_{2}^{j} x_{3}^{m(i, j)-2} .
\end{aligned}
$$

Clearly, $\mathbf{x}$ is an eigenvector of $R$ with eigenvalue $m(m-1) \lambda$. Then, by optimization theory and [24], if the other two eigenvalues of $R$ are less than $m(m-1) \lambda$, then $\mathbf{x}$ is a local maximizer of (18). On the other hand, if $\mathbf{x}$ is a local maximizer of (18), then the other two eigenvalues of $R$ are less than or equal to $m(m-1) \lambda$.

Suppose that $\mathbf{x}$ is a local maximizer of (16) with Zeigenvalue $\lambda$. If $\lambda$ is not larger than one of the other $Z$ eigenvalues which corresponds to saddle points or local minimizers, then such a direction is not significant. In view of this, a Z-eigenvalue of $u$ is called a principal Z-eigenvalue if it is dominates all the other eigenvalues which corresponds to saddle points or local minimizers. In this way, a Z-eigenvector of $u$, associated with a principal Z-eigenvalue, is regarded as a principal direction. An illustration for the principal direction of ODF is given in Fig. 1.

\section{Experimental Results}

In this section, we report some experimental results on our method applied to simulated dataset as well as real human
Fig. 1 Illustration for the principal directions of ODF, where the red line denotes the maximum principal direction, the blue one is the second principal direction, and the black one is the third principal direction (Color figure online)

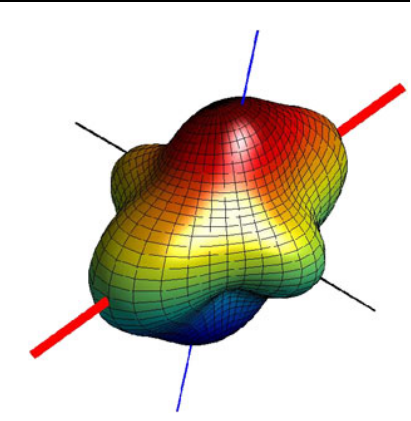

brain dataset. Firstly, we generate synthetic HARDI data by the following multilinear model [1]:

$S\left(g_{i}\right)=\sum_{k=1}^{n} p_{k} e^{-b g_{i} D_{k} g_{i}}+$ noise,

where $n \in\{0,1,2,3\}$ is the number of fibers, $p_{k}$ is the proportion of tissue in the voxel that corresponds to the $k$ th fiber $\left(\sum_{k=1}^{n} p_{k}=1\right), b$ is the $b$-value, $g_{i}$ is the $i$ th gradient direction for $i \in\{1, \ldots, 81\}$, and $D_{k}$ is the diffusion tensor of the $k$ th fiber. This synthetic data generation is relatively standard and has advantage of analytic computation of the ODF [11]. The noise was typically generated by Rician noise (complex Gaussian noise) with standard deviation of $1 / \sigma$, producing a signal to noise ratio (SNR) of $\sigma$. In our experiments, unless special instructions, the $b$ value equals to $3000 \mathrm{~s} / \mathrm{mm}^{2}$ and the diffusion tensors were selected such as $D_{k}=\operatorname{diag}(1700,200,200) \times 10^{-6} \mathrm{~mm}^{2} / \mathrm{s}$ for $k=1,2,3$. And we generated Rician-corrupted data $S$ as done in [12]. For each noise-free data $x$, we computed $S$ as:

$S=\sqrt{\left(\frac{x}{\sqrt{2}}+n_{r}\right)^{2}+\left(\frac{x}{\sqrt{2}}+n_{i}\right)^{2}}$

where $n_{r}$ and $n_{i} \sim \mathcal{N}\left(0, \sigma^{2}\right)$. The value $S$ is the realisation of a random variable with a Rician p.d.f. of parameters $x$ and $\sigma$.

Firstly, we demonstrate qualitatively that our method can guarantee nonnegative diffusivity by comparing it with the Least Squares (LS) method. The LS method is a simple approach which estimates the coefficients of an ODF function. This method is usually easily implementable and fast. On the other hand, it does not guarantee positive diffusivity. Below, we estimate the ODF fitting with a 4th order diffusion tensor in the homogeneous polynomial basis using these two algorithms. For the single tensor model, the ODF function (the SNR was fixed to 35) estimated by the LS method, fitting with a 4th order tensor, is $\operatorname{ODF}(x)=u^{T} \hat{x}$, where $u$ is a 15dim vector with $u(1)=-0.7344, u(2)=-0.0010, u(3)=$ $1.7255, u(4)=-0.0140, u(5)=-0.6048, u(6)=0.0142$, $u(7)=0.0055, u(8)=0.0203, u(9)=-0.0096, u(10)=$ $-0.1701, u(11)=0.0035, u(12)=-0.2132, u(13)=$ $-0.0650, u(14)=-0.0035, u(15)=7.1234$. Using the 
Table 1 Z-eigenvalues and eigenvectors of a 4th order tensor, where the ODF was estimated by LS method

\begin{tabular}{rrrlr}
\hline & \multicolumn{1}{c}{$x_{1}$} & \multicolumn{1}{l}{$x_{2}$} & \multicolumn{1}{l}{$x_{3}$} & \multicolumn{1}{l}{$\lambda$} \\
\hline 1 & -0.0055 & 0.0002 & 1.0000 & -0.7344 \\
2 & 0.0048 & 1.0000 & 0.0024 & -0.6048 \\
3 & -0.1634 & 0.7117 & 0.6832 & 0.0878 \\
4 & 0.1559 & 0.7119 & 0.6847 & 0.0906 \\
5 & 0.0137 & 0.7211 & 0.6927 & 0.0941 \\
6 & -0.1689 & -0.7120 & 0.6816 & 0.0945 \\
7 & 0.1577 & -0.7135 & 0.6826 & 0.0985 \\
8 & 0.0174 & -0.7225 & 0.6911 & 0.1020 \\
\hline
\end{tabular}

Table 2 Z-eigenvalues and eigenvectors of a 4th order tensor, where the ODF was estimated by PSD method

\begin{tabular}{rrrll}
\hline & \multicolumn{1}{c}{$g_{1}$} & \multicolumn{1}{l}{$g_{2}$} & \multicolumn{1}{l}{$g_{3}$} & $\lambda$ \\
\hline 1 & -0.0023 & 0.0001 & 1.0000 & 0.0001 \\
2 & 0.0017 & 1.0000 & 0.0015 & 0.1297 \\
3 & -0.0100 & 0.7160 & 0.6980 & 1.2786 \\
4 & -0.0135 & -0.7168 & 0.6971 & 1.2862 \\
\hline
\end{tabular}

method provided in Appendix, we can compute all the Zeigenvalues and the associated eigenvectors, which are listed in Table 1.

From Table 1, we can see that there are two negative eigenvalues and the smallest Z-eigenvalue is -0.7344 , attained at $(-0.0055,0.0002,1.0000)$. This shows the need for enforcing the positive semi-definite property of the estimated tensor since negative diffusivity profiles are not meaningful from the point of view of physics.

Next, we illustrate that our method can guarantee positive diffusivity. In the same example above, the ODF function estimated by the our method is $O D F(x)=u^{T} \hat{x}$, with $u(1)=$ $0.0001, u(2)=-0.001, u(3)=4.9965, u(4)=-0.014$, $u(5)=0.1297, u(6)=0.0142, u(7)=0.0055, u(8)=$ $0.0203, u(9)=-0.0096, u(10)=3.1009, u(11)=0.0035$, $u(12)=3.0578, u(13)=-0.065, u(14)=-0.0035$, $u(15)=7.8579$. All Z-eigenvalues and the associated eigenvectors were listed in Table 2 . As we see that the smallest $Z$ eigenvalue is 0.0001 , attained at $(-0.0023,0.0001,1.0000)$.

We also borrowed a picture from [19] to illustrate effects of the approach with guaranteed nonnegative diffusivity. If a final ODF estimation contains negative diffusivity, it will result in negative side lobes as indicated in black in Fig. 2. Meanwhile, the approach with guaranteed nonnegative diffusivity can eliminate these side lobes completely.

Next, in order to compare the robustness of our method in the presence of noise, we generated the signals by (17) at 5 different SNR ranging from 10 to 50 and repeated the experiments 10 times. We estimate the ODF fitting with 6th order diffusion tensor. We choose to compare our PSD method
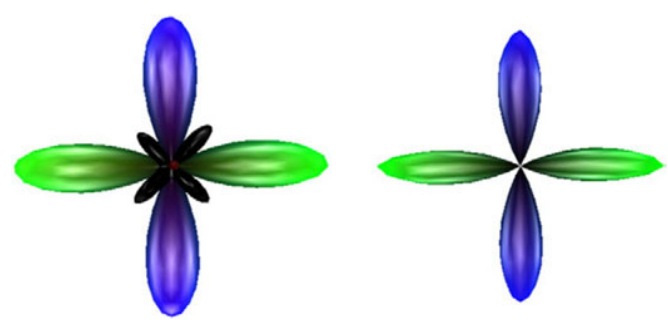

Fig. 2 (Borrowed from [19] to illustrate effects of the approach with guaranteed nonnegative diffusivity.) Without projection (left), the final ODF estimation contains negative-valued regions (the side lobes in black). Meanwhile, enabling projection results in elimination of these negative side lobes (right)

against the following two methods: (1) a nonnegative constrained Least Squares (LS+) method: solve (4) and by using the linear transformation to get $\bar{u}=A \bar{s}$, then set negative ODF as zero, i.e. project it onto the nonnegative space, finally get the solution $u=\mathcal{P}^{+}(\bar{u})$; (2) and a constrained spherical deconvolution (CSD) method [29]. For the CSD method, a constraint is introduced to minimize the appearance of negative values in the reconstructed FOD. But it does not completely forbid negative FOD values. We implemented the CSD method with $\lambda=1$ and $\tau=10 \%$ of the mean initial FOD amplitude (see [29] for a detailed description of these parameters). Then, we computed the means of angles errors in degree between the actual fiber orientations and the maxima of estimated ODF/FOD. The results are plotted in Fig. 3. As would be expected the means of the degree error decreases as the SNR increases. The PSD method compares favorably to the least squares (LS+) method. Comparing with the PSD method, the CSD method gains an improvement of approximately $1^{\circ}$. Figure 4 also shows the precision of PSD method in the presence of varying levels of noise, for different $b$-values. The red line is for $b=1000$, while black is for $b=3000$. As we can see from Fig. 4, when $S N R=50$, the angular error is about $3.3^{\circ}$ at $b=1000$ while it is about $2.4^{\circ}$ at $b=3000$.

Next we worked on a phantom dataset [20], which was acquired on a GE Healcare Signa 1.5 T scanner. It had 4000 gradient directions and for our experiments we used a bvalue of $4000 \mathrm{~s} / \mathrm{mm}^{2}$. The phantom had a geometry of two fiber bundles crossing perpendicularly close to $\mathrm{X}$-axis and the Y-axis. Figure 5 shows the ODF profiles. We estimated the 2nd order diffusion tensor (shown in the top) and the 4th order diffusion tensor using our method. The extracted principle direction of ODF indicate the known fiber geometry of the phantom. These results also demonstrate that high order tensor estimation is necessary since 2 nd order diffusion tensor fails to approximate complex local tissue structure.

In the next experiment, we are interested to estimate the ODF profiles from human brain dataset with size of $90 \times 90 \times 60$, which was acquired on a $1.5 \mathrm{~T}$ scanner at $b=1000 \mathrm{~s} / \mathrm{mm}^{2}$ using 60 encoding directions, with voxel 
Fig. 3 Effects of varying SNR on the detected maximum of the ODF estimations by LS+ method, CSD method and our positive semi-definite (PSD) approach for $b=3000$

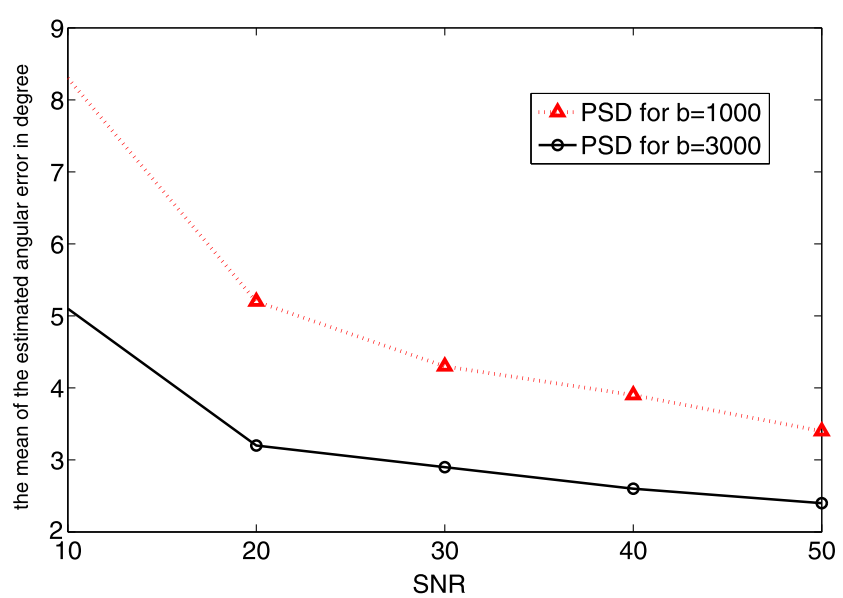

Fig. 4 Effects of varying SNR on the detected maximum of the ODF estimations by PSD method for different $b$-values. The red line is for $b=1000$, while black is for $b=3000$. The $y$-axis is the mean of the estimated angular error (Color figure online)

dimensions of $1.875 \mathrm{~mm} \times 1.875 \mathrm{~mm} \times 2 \mathrm{~mm}$. In this experiment we estimate the analytical ODF in the SH basis with rank-4 from this HARDI signal by MATLAB 7.4. We show some reconstructed ODFs with sharpening in Fig. 6, in which the parameter $\alpha=0.006$. We also show some reconstructed ODFs with principal directions in the region of interest (ROI). We can detect there are multiple fibers in which some crossing are due to diverging or splitting fibers. These results show that our nonnegative ODF profiles model can depict the characterization of diffusion anisotropy which was consistent with known neuroanatomy.

\section{Conclusion}

In conclusions, the main contribution of this paper is of threefold:

- Firstly, we showed that there is a constant linear transformation relation between the vector versions of the raw

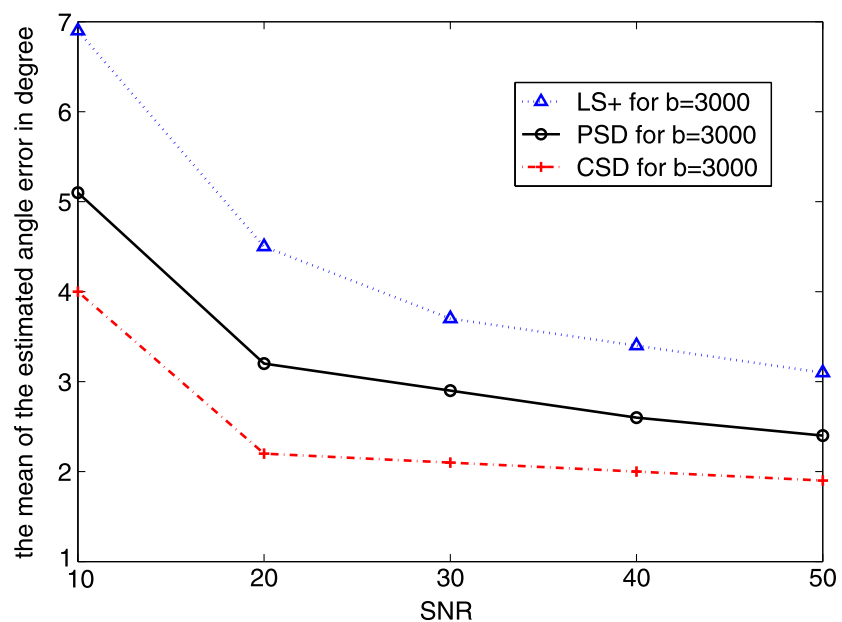

HARDI signal and the ODF in the homogeneous polynomial basis. Such a linear transformation connection enable us to model the nonnegative feature of the ODF.

- Secondly, we proposed a new reconstruction framework to estimate nonnegative ODFs from HARDI data. Interesting features of this model include minimizing a convex optimization problem with a convex quadratic objective function constrained by the nonnegativity requirement on the smallest Z-eigenvalue of the diffusivity tensor. This model can guarantee the positive semi-definite property of the estimated high order tensor (not limited to 4th order tensor), which is the main contribution of our work. This property is essential since negative diffusivity profiles are not meaningful from the point of view of physics.

- Thirdly, based on optimization theory and techniques, we present a computational method for determining the principal directions of the ODF. Numerical examples on synthetic data as well as MRI data are tested to illustrate the significance of our approach.

It would be interesting to see how we can extend our nonnegative ODF model to tackle tractography. Moreover, we also would like to see how our model performs if we use it to analyze in real datasets at $b=3000$ (or higher $b$-values) where fiber crossings could be better detected. These will be our future research directions.

Acknowledgements The authors would like to acknowledge Maxime Descoteaux and Rachid Deriche and the Odyssee Diffusion MRI Toolbox. The authors would also like to thank Cyril Poupon for his kind offer of the diffusion phantom dataset. The authors would also like to thank three anonymous referees who have contributed to improve the quality of the paper.

This work was partly supported by the Research Grant Council of Hong Kong and the National Natural Science Foundation of China (No. 11001060), the Natural Science Foundation of Jiangxi Province, China (2009GQS0007) and the program of JGZX (20112BCB23027). 

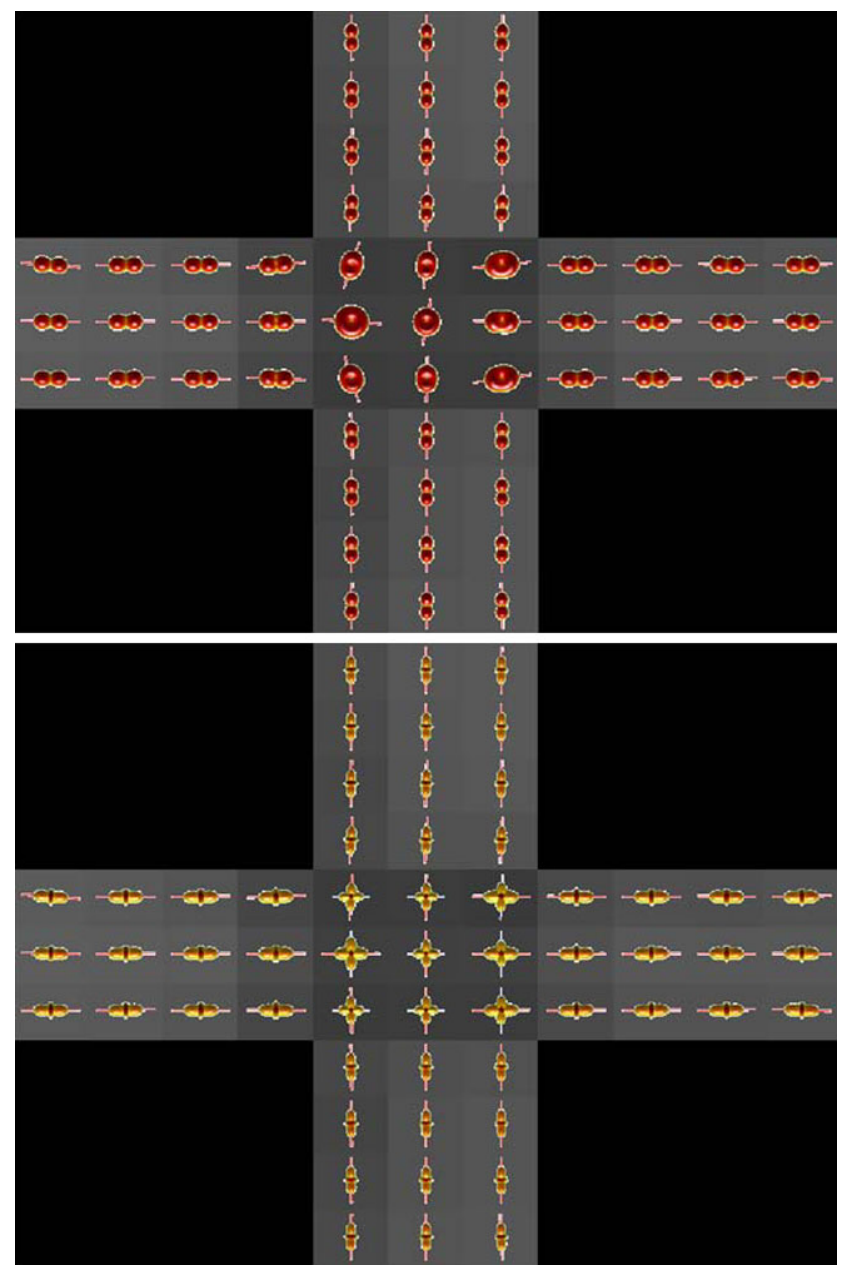

Fig. 5 ODF profile from a Phantom dataset. The phantom had a geometry of two fiber bundles crossing perpendicularly close to the $X$-axis and the $Y$-axis. We estimated the 2nd order diffusion tensor (shown in the top) and the 4th order diffusion tensor (shown in the bottom) using our method. As we can see for the 4th order diffusion tensor, the extracted principle direction of ODF could indicate the known fiber geometry of the phantom

\section{Appendix: The Solution Method for (7)}

According to optimization theory, the optimality conditions of (7) have the form:

$$
\left\{\begin{array}{l}
\sum_{i=1}^{m} \sum_{j=0}^{m-i} i b_{i j} x_{1}^{i-1} x_{2}^{j} x_{3}^{m(i, j)}=m \lambda x_{1}, \\
\sum_{i=0}^{m} \sum_{j=1}^{m-i} j b_{i j} x_{1}^{i} x_{2}^{j-1} x_{3}^{m(i, j)}=m \lambda x_{2}, \\
\sum_{i=0}^{m} \sum_{j=0}^{m-i-1} m(i, j) b_{i j} x_{1}^{i} x_{2}^{j} x_{3}^{m(i, j)-1}=m \lambda x_{3}, \\
x_{1}^{2}+x_{2}^{2}+x_{3}^{2}=1 .
\end{array}\right.
$$

Here $m(i, j)=m-i-j$. The additional " $m$ " on the right hand sides of the first three equations make it the same as the definition of Z-eigenvalues [7, 21-24] for the symmetric tensor $x$. If $(\mathbf{x}, \lambda)$ is a solution of (18), then $\mathbf{x}$ is a stationary point of (7) and

$\lambda=\Psi(\mathbf{x})$

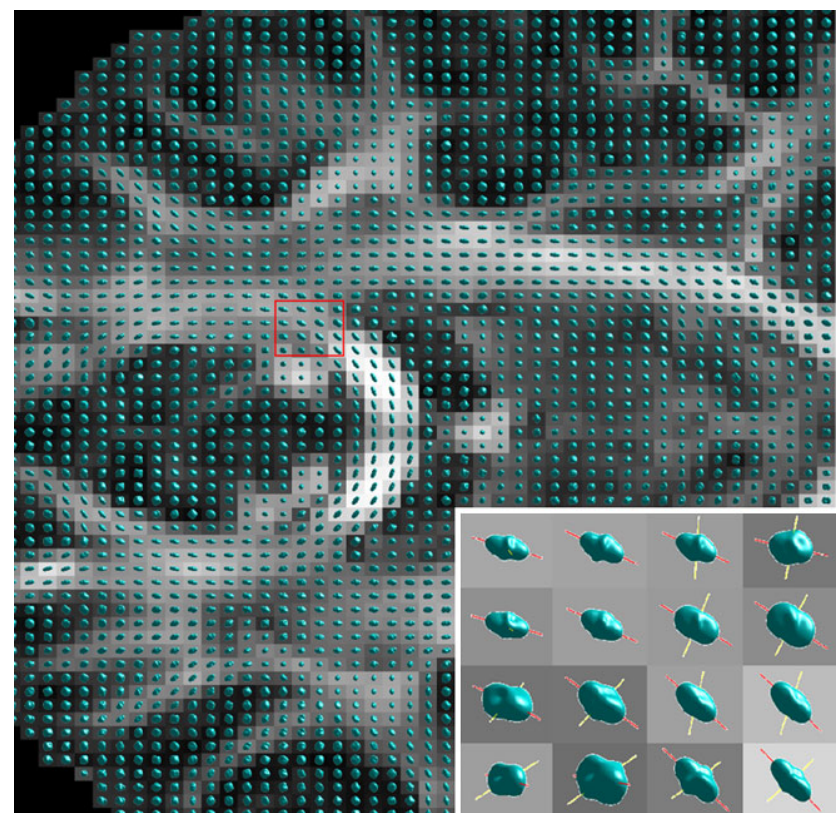

Fig. 6 Reconstructed ODFs from human brain with sharpening, shown on the Generalized Fractional Anisotropy (GFA) map, where GFA was defined by Tuch [27] as GFA $=\frac{s t d(O D F)}{r m s(O D F)}$. We also reconstructed ODFs with principal directions for a region of interest (shown in the bottom). We can detect there are multiple fibers in which some crossing are due to diverging or splitting fibers

is a Z-eigenvalue of $u$. Then, the smallest Z-eigenvalue of $u$ is the optimal value of (7).

We may solve (18) in the following way:

Case $1 x_{3}=x_{2}=0$. By (18), this only happens if $b_{m-1,1}=$ $b_{m-1,0}=0$. In this case, $x_{1}= \pm 1, \lambda=b_{m, 0}$.

Case $2 x_{3}=x_{1}=0$. By (18), this only happens if $b_{1, m-1}=$ $b_{0, m-1}=0$. In this case, $x_{2}= \pm 1, \lambda=b_{0, m}$.

Case $3 x_{3}=0, x_{1} \neq 0$ and $x_{2} \neq 0$. Then (18) becomes

$\left\{\begin{array}{l}\sum_{i=1}^{m} i b_{i, m-i} x_{1}^{i-1} x_{2}^{m-i}=m \lambda x_{1}, \\ \sum_{i=0}^{m-1}(m-i) b_{i, m-i} x_{1}^{i} x_{2}^{m-i-1}=m \lambda x_{2}, \\ \sum_{i=0}^{m-1} b_{i, m-i-1} x_{1}^{i} x_{2}^{m-i-1}=0, \\ x_{1}^{2}+x_{2}^{2}=1 .\end{array}\right.$

We may eliminate $\lambda$ in (20) and have the following equations of $x_{1}$ and $x_{2}$ :

$\left\{\begin{array}{l}\sum_{i=1}^{m} i b_{i, m-i} x_{1}^{i-1} x_{2}^{m-i+1} \\ \quad=\sum_{i=0}^{m-1}(m-i) b_{i, m-i} x_{1}^{i+1} x_{2}^{m-i-1}, \\ \sum_{i=0}^{m-1} b_{i, m-i-1} x_{1}^{i} x_{2}^{m-i-1}=0, \\ x_{1}^{2}+x_{2}^{2}=1 .\end{array}\right.$ 
Let $t=x_{1} / x_{2}$. We have

$\left\{\begin{array}{l}\sum_{i=1}^{m} i b_{i, m-i} t^{i-1}=\sum_{i=0}^{m-1}(m-i) b_{i, m-i} t^{i+1}, \\ \sum_{i=0}^{m-1} b_{i, m-i-1} t^{i}=0 .\end{array}\right.$

We may solve the two one-variable equations of (21) separately. If they have common solutions $t$, then (18) has solutions

$x_{1}=\frac{t}{\sqrt{1+t^{2}}}, \quad x_{2}=\frac{ \pm 1}{\sqrt{1+t^{2}}}$,

$x_{3}=0, \quad \lambda=\Psi(\mathbf{x})$.

Case $4 x_{3} \neq 0$. We may eliminate $\lambda$ in (18) and have the following equations of $\mathbf{x}$ :

$$
\left\{\begin{array}{l}
\sum_{i=1}^{m} \sum_{j=0}^{m-i} i b_{i j} x_{1}^{i-1} x_{2}^{j} x_{3}^{m(i, j)+1} \\
\quad=\sum_{i=0}^{m} \sum_{j=0}^{m-i-1} m(i, j) b_{i j} x_{1}^{i+1} x_{2}^{j} x_{3}^{m(i, j)-1} \\
\sum_{i=0}^{m} \sum_{j=1}^{m-i} j b_{i j} x_{1}^{i} x_{2}^{j-1} x_{3}^{m(i, j)+1} \\
\quad=\sum_{i=0}^{m} \sum_{j=0}^{m-i-1} m(i, j) b_{i j} x_{1}^{i} x_{2}^{j+1} x_{3}^{m(i, j)-1} \\
x_{1}^{2}+x_{2}^{2}+x_{3}^{2}=1
\end{array}\right.
$$

Let $w=x_{1} / x_{3}, v=x_{2} / x_{3}$. Then we have

$$
\left\{\begin{array}{l}
\sum_{i=1}^{m} \sum_{j=0}^{m-i} i b_{i j} w^{i-1} v^{j} \\
\quad=\sum_{i=0}^{m} \sum_{j=0}^{m-i-1} m(i, j) b_{i j} w^{i+1} v^{j}, \\
\sum_{i=0}^{m} \sum_{j=1}^{m-i} j b_{i j} w^{i} v^{j-1} \\
\quad=\sum_{i=0}^{m} \sum_{j=0}^{m-i-1} m(i, j) b_{i j} w^{i} v^{j+1} .
\end{array}\right.
$$

For solving system (23), we first regard it as a system of polynomial equations of variable $w$ and rewrite it as

$\left\{\begin{array}{l}\gamma_{0} w^{m}+\gamma_{1} w^{m-1}+\cdots+\gamma_{m}=0, \\ \tau_{0} w^{m-1}+\tau_{1} w^{m-2}+\cdots+\tau_{m-1}=0,\end{array}\right.$

where $\gamma_{0}, \ldots, \gamma_{m}, \tau_{0}, \ldots, \tau_{m-1}$ are polynomials of $v$, which can be calculated by (19). By the Sylvester theorem, the above system of polynomial equations in $w$ possesses solutions if and only if its resultant vanishes [9]. The resultant of this system of polynomial equations is the determinant of the following $(2 m-1) \times(2 m-1)$ matrix

$$
V:=\left(\begin{array}{ccccccccc}
\gamma_{0} & \gamma_{1} & \cdots & \gamma_{m-2} & \gamma_{m-1} & \gamma_{m} & \cdots & 0 & 0 \\
0 & \gamma_{0} & \cdots & \gamma_{m-3} & \gamma_{m-2} & \gamma_{m-1} & \cdots & 0 & 0 \\
. & \cdot & \cdots & . & . & . & \cdots & . & . \\
0 & 0 & \cdots & \gamma_{1} & \gamma_{2} & \gamma_{3} & \cdots & \gamma_{m} & 0 \\
0 & 0 & \cdots & \gamma_{0} & \gamma_{1} & \gamma_{2} & \cdots & \gamma_{m-1} & \gamma_{m} \\
\tau_{0} & \tau_{1} & \cdots & \tau_{m-2} & \tau_{m-1} & 0 & \cdots & 0 & 0 \\
0 & \tau_{0} & \cdots & \tau_{m-3} & \tau_{m-2} & \tau_{m-1} & \cdots & 0 & 0 \\
. & . & \cdots & . & . & . & \cdots & . & . \\
0 & 0 & \cdots & \tau_{0} & \tau_{1} & \tau_{2} & \cdots & \tau_{m-1} & 0 \\
0 & 0 & \cdots & 0 & \tau_{0} & \tau_{1} & \cdots & \tau_{m-2} & \tau_{m-1}
\end{array}\right),
$$

which is a polynomial equation in variable $v$. After finding all real roots of this polynomial, we can substitute them to
(23) to find all the real solutions of $w$. Then, using $x_{1}=$ $\frac{w}{\sqrt{1+w^{2}+v^{2}}}, x_{2}=\frac{v}{\sqrt{1+w^{2}+v^{2}}}, x_{3}=\frac{ \pm 1}{\sqrt{1+w^{2}+v^{2}}}, \lambda=\Psi(\mathbf{x})$, we may find all the solutions of (18) in this case.

Combine all the possible solutions of (18) in these four cases, and find $\lambda_{\min }(A s)$, the smallest value of $\lambda$ of these solutions. This solves (7).

\section{References}

1. Alexander, D.C., Barker, G.J., Arridge, S.R.: Detection and modeling of non-Gaussian apparent diffusion coefficient profiles in human brain data. Magn. Reson. Med. 48, 331-340 (2002)

2. Barmpoutis, A., Hwang, M.S., Howland, D., Forder, J.R., Vemuri, B.C.: Regularized positive-definite fourth order tensor filed estimation from DW-MRI. NeuroImage 45, 5153-5162 (2009)

3. Barmpoutis, A., Jian, B., Vemuri, B.C., Shepherd, T.M.: Symmetric positive 4th order tensors \& their estimation from diffusion weighted MRI. In: Karssemeijer, M., Lelieveldt, B. (eds.) Information Processing and Medical Imaging, pp. 308-319. Springer, Berlin (2007)

4. Basser, P.J., Jones, D.K.: Diffusion-tensor MRI: theory, experimental design and data analysis-a technical review. NMR Biomed. 15, 456-467 (2002)

5. Basser, P.J., Mattiello, J., LeBihan, D.: Estimation of the effective self-diffusion tensor from the NMR spin echo. J. Magn. Reson., Ser. B 103, 247-254 (1994)

6. Basser, P.J., Mattiello, J., LeBihan, D.: MR diffusion tensor spectroscopy and imaging. Biophysica 66, 259-267 (1994)

7. Bloy, L., Verma, R.: On computing the underlying fiber directions from the diffusion orientation distribution function. In: Metaxas, D., Axel, L., Fichtinger, G., Székeley, G. (eds.) Medical Image Computing and Computer-Assisted Intervention-MICCAI 2008, pp. 1-8. Springer, Berlin (2008)

8. Chefd'Hotel, C., Tschumperle, D., Deriche, R., Faugeras, O.: Regularizing flows for constrained matrix-valued images. J. Math. Imaging Vis. 20, 147-162 (2004)

9. Cox, D., Little, J., O'Shea, D.: Using Algebraic Geometry. Springer, New York (1998)

10. Descoteaux, M., Angelino, E., Fitzgibbons, S., Deriche, R.: Apparent diffusion coefficients from high angular diffusion imaging: estimation and applications. Magn. Reson. Med. 56, 395-410 (2006)

11. Descoteaux, M., Angelino, E., Fitzgibbons, S., Deriche, R.: Regularized, fast, and analytical q-ball imaging. Magn. Reson. Med. 58, 497-510 (2007)

12. Descoteaux, M., Wiest-Daesslé, N., Prima, S., Barillot, C., Deriche, R.: Impact of Rician adapted non-local means filtering on HARDI. In: MICCAI 2008, Part II. LNCS, vol. 5242, pp. 122130 (2008)

13. Ghosh, A., Deriche, R.: From second to higher order tensors in diffusion-MRI. In: Tensors in Image Processing and Computer Vision, pp. 315-334. Springer, London (2009)

14. Ghosh, A., Tsigaridas, E., Descoteaux, M., Comon, P., Mourrain, B., Deriche, R.: A polynomial based approach to extract the maxima of an antipodally symmetric spherical function and its application to extract directions from the orientation distribution function in diffusion MRI. In: Workshop on Computational Diffusion MRI, Held in Conjunction with the MICCAI 2008 Conference, New York, USA, September 2008 (2008)

15. Ghosh, A., Descoteaux, M., Deriche, R.: Riemannian framework for estimating symmetric positive definite 4th order diffusion tensors. In: Metaxas, D., Axel, L., Fichtinger, G., Székeley, G. (eds.) Medical Image Computing and Computer-Assisted InterventionMICCAI 2008, pp. 858-865. Springer, Berlin (2008) 
16. Hiriart-Urruty, J.-B., Lemaréchal, C.: Convex Analysis and Minimization Algorithms. Springer, Berlin (1993)

17. Nocedal, J., Wright, S.J.: Numerical Optimization. Springer, New York (1999)

18. Ozarslan, E., Mareci, T.H.: Generalized diffusion tensor imaging and analytical relationships between diffusion tensor imaging and high angular resolution diffusion imaging. Magn. Reson. Med. 50, 955-965 (2003)

19. Patel, V., Shi, Y., Thompson, P.M., Toga, A.W.: Mesh-based spherical deconvolution: a flexible approach to reconstruction of nonnegative fiber orientation distributions. NeuroImage 51, 10711081 (2010)

20. Poupon, C., Rieul, B., Kezele, I., Perrin, M., Poupon, F., Mangin, J.F.: New diffusion phantoms dedicated to the study and validation of HARDI models. Magn. Reson. Med. 60, 76-83 (2008)

21. Qi, L.: Eigenvalues of a real supersymmetric tensor. J. Symb. Comput. 40, 1302-1324 (2005)

22. Qi, L., Wang, Y., Wu, E.X.: D-eigenvalues of diffusion kurtosis tensors. J. Comput. Appl. Math. 221, 150-157 (2008)

23. Qi, L., Han, D., Wu, E.X.: Principal invariants and inherent parameters of diffusion kurtosis tensors. J. Math. Anal. Appl. 349, 165-180 (2009)

24. Qi, L., Wang, F., Wang, Y.: Z-eigenvalue methods for a global polynomial optimization problem. Math. Program. 118, 301-316 (2009)

25. Qi, L., Yu, G., Wu, E.X.: Higher order positive semi-definite diffusion tensor imaging. SIAM J. Imaging Sci. 3, 416-433 (2010)

26. Rockafellar, R.T.: Convex Analysis. Princeton Publisher, Princeton (1970)

27. Tuch, D.S.: Q-ball imaging. Magn. Reson. Med. 52, 1358-1372 (2004)

28. Tuch, D.S., Reese, T.G., Wiegell, M.R., Makris, N.G., Belliveau, J.W., Wedeen, V.J.: High angular resolution diffusion imaging reveals intravoxel white matter fiber heterogeneity. Magn. Reson. Med. 48, 454-459 (2002)

29. Tournier, J.-D., Calamante, F., Connelly, A.: Robust determination of the fibre orientation distribution in diffusion MRI: Non-negativity constrained super-resolved spherical deconvolution. NeuroImage 35, 1459-1472 (2007)

30. Wang, Z., Vemuri, B.C., Chen, Y., Mareci, T.H.: A constrained variational principle for direct estimation and smoothing of the diffusion tensor field from complex DWI. IEEE Trans. Med. Imaging 23, 930-939 (2004)

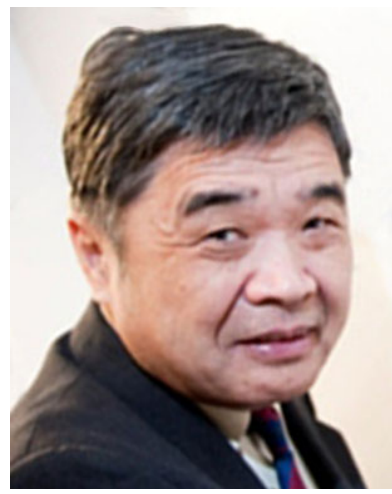

Liqun Qi received his B.S. in Computational Mathematics at Tsinghua University in 1968, his M.S, and Ph.D. degree in Computer Sciences at University of Wisconsin-Madison in 1981 and 1984, respectively. Professor Qi has taught in Tsinghua University, China, University of Wisconsin- Madison, USA, University of New South Wales, Australia, and The City University of Hong Kong. He is now Chair Professor of Applied Mathematics and Head of Department of Applied Mathe- matics at The Hong Kong Polytechnic University. Professor Qi has published more than 200 research papers in international journals. $\mathrm{He}$ established the superlinear and quadratic convergence theory of the semismooth Newton method, and played a principal role in the development of reformulation methods in optimization. Professor Qi's research work has been cited by the researchers around the world. According to the authoritative citation database www.isihighlycited.com, he is one of the world's most highly cited 300 mathematicians. Professor Qi is an editor or an associate editor of ten international journals. He has chaired more than ten international conferences and workshops held at Australia, Italy, Hong Kong and the Mainland China. In 2005, Professor Qi pioneered the research on eigenvalues for higher order tensors, which now has applications in biomedical engineering, statistical data analysis, spectral hypergraph theory, solid mechanics, etc. Professor Qi played a leading role in the optimization community in the Asian Pacific region. In 2000, with five other optimization researchers in this region together, Professor Qi initiated the Pacific Optimization Research Activity Group and the Sino-Japanese Optimization Meeting Series. Professor Qi also works closely with researchers in the Mainland China. In 2010, Professor Qi received the First Class Science and Technology Award of Chinese Operations Research Society.

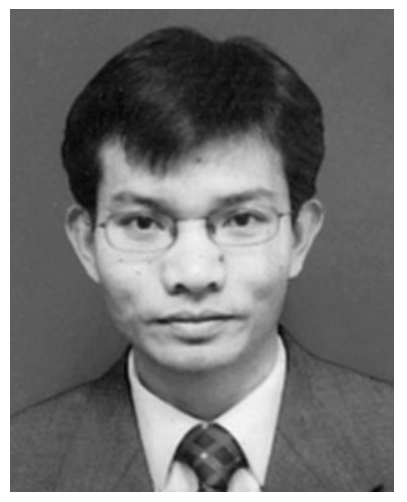

Gaohang Yu received his Ph.D. degree in Computational Mathematics at Sun Yat-Sen University in 2007. From 2007 to 2009, he taught in Sun Yat-Sen University. Then he joined the School of Mathematics and Computer Science at the Gannan Normal University as an Associate Professor. From 2008 to 2011, he ever worked at Department of Applied Mathematics at The Hong Kong Polytechnic University as a postdoctoral/research fellow. His research interests include optimization theory and methods, image processing, and medical imaging.

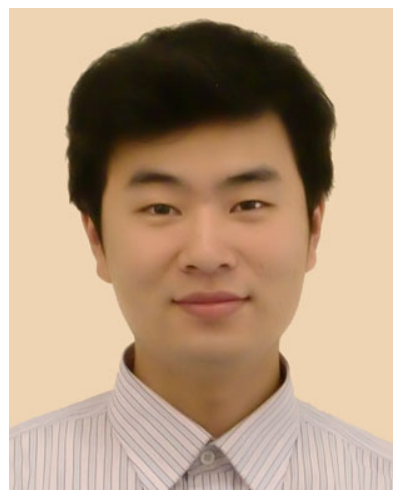

Yi Xu is a Ph.D. candidate at Department of Applied Mathematics at The Hong Kong Polytechnic University; he received his B.S. at Nanjing Normal University in 2005. His research interests include numerical optimization and applications. 